\title{
Integrated diffractive shearing interferometry for adaptive wavefront sensing
}

\author{
Jason H. Karp, ${ }^{*}$ Trevor K. Chan, and Joseph E. Ford \\ Department of Electrical and Computer Engineering, University of California, \\ San Diego, 9500 Gilman Drive, La Jolla, California 92093-0407, USA \\ ${ }^{*}$ Corresponding author: jkarp@ucsd.edu
}

Received 3 July 2008; revised 1 October 2008; accepted 4 November 2008; posted 6 November 2008 (Doc. ID 98299); published 0 MONTH 0000

\begin{abstract}
We present theory, design, and preliminary experimental studies for a compact wavefront sensor based on lateral shearing interferometry using a binary phase grating, image sensor, and Fourier-based processing. The integrated system places a diffractive element directly onto an image sensor to generate interference fringes within overlapping diffraction orders. The shearing ratio and the interferogram signal-to-noise ratio directly affect the reconstruction accuracy of wavefronts with differing spatial variations. Optimal shearing parameters associated with the autocorrelation of the input encourage placing a spatial light modulator as the diffractive element allowing adaptive wavefront sensing. Experimental results from a fixed-grating system are presented as well as requirements for next-generation adaptive systems. (C) 2008 Optical Society of America

OCIS codes: $\quad 070.6120,110.3175,130.3120$
\end{abstract}

\section{Introduction}

Wavefront sensing describes phase-sensitive detection from optical path differences (OPDs) within a field. Optical wavefront sensors are used in metrology applications ranging from corrective vision surgery to adaptive free-space communication. All wavefront sensors map phase information into intensity variations for detection. Several types of system exist based on their mapping techniques. Phase shifting interferometry forms a linear intensity pattern on the object surface and scans through a set phase shifts to visualize the OPD [1]. Shack-Hartmann $(\mathrm{SH})$ sensors place a lens array above an image sensor, resulting in focal-spot position shifts relating to local wavefront tilt [2]. Lateral shearing interferometry (LSI) overlaps a wavefront with shifted replicas of itself, creating an interferogram with differential phase contours.

SH sensors have gained significant attention in the past decade owing to their compact and integrated form factor that lends itself well to practical

0003-6935/08/360001-01\$15.00/0

(C) 2008 Optical Society of America devices. Commercial Hartmann masks consist of a two-dimensional array of lenses to sample the incoming wavefront and focus onto a high-resolution image sensor. Aberrations such as coma, spherical and astigmatism, as well as tilt and defocus can be calculated from the spot field and are especially useful in adaptive-optic feedback loops. However, one must assume the maximum tilt and spatial frequency of the aberration when selecting the lenslet and detector parameters. Spatial variations smaller than the lens diameter are not sampled. Large local tilts form focal spots behind adjacent lenses, leading to identification errors [2]. SH devices can be sensitive to over $\lambda / 20$ but place an upper bound on the dynamic range of the wavefront due to the trade-off defined by the sensor and lens selection.

Optical interferometry offers exceptional sensitivity relating to the short wavelength of visible or infrared light. Twyman-Green and Fizeau interferometers are commonly used in astronomy and aberrometry along with deformable mirrors to compensate for atmospheric distortions. Traditional interferometers such as these require strict alignment and vibration isolation to produce stable 
fringes, since a reference arm is needed to interfere with the aberrated wavefront arm [3]. Ronchi first proposed the use of linear patterns imaged onto mirrors to observe phase distortions [4], and this technique is the basis for using LSI as a wavefront sensor. LSI generates an interferogram by overlapping copies of an identical wavefront with induced lateral shift and/or tilt. Michelson, Mach-Zehnder, and Sagnac interferometers can all be designed for lateral shearing; however, our focus is on diffractive shearing $[5,6]$. The self-referenced design inherent to LSI removes vibration and isolation concerns, since no planar reference arm is used. This is also a drawback. The interferogram embeds phase information between two unknown signals, thereby requiring postprocessing and reconstruction algorithms. Despite the added computation, adjustable shear magnitude is a degree of freedom inherent to LSI and is not found in other sensing techniques. This parameter can provide varied levels of sensitivity based on the type and severity of the input aberration, allowing LSI to potentially surpass limitations restricting other wavefront sensing modalities.

\section{Integrated Diffractive Shearing}

Integrated wavefront sensors are finding uses in a broad range of new applications because of their ability to stand alone as a complete system. An integrated system refers to a compact optical setup, including all devices and sensors, aligned and mounted in an all-inclusive package. SH devices are excellent examples of integrated wavefront sensors; but sensitivity trade-offs lead to reduced resolution at certain wavefront aberrations. Our goal in this work is to explore the design and limitations of a LSI diffractive wavefront sensor with adjustable sensitivity to accommodate a wide range of profiles.

LSI first creates replicas of an unknown wavefront and overlaps them with some shift and/or tilt to form an interference pattern within the overlapping area. The contours associated with the fringe pattern differ significantly as the lateral shear distance changes, because no known reference phase front is present. Parallel plate surface reflections and grating diffraction orders are two methods for generating the overlapping beam replicas needed for LSI [5]. Gratings can have very high diffraction efficiencies, thereby using the illumination effectively compared with surface reflections, and gratings remain the focus of this work.

Interference fringes occur only in the area of overlap, directly relating to the OPD between the points of intersection. Measuring a two-dimensional field requires two nonparallel shears, often performed in orthogonal directions. Fig. 1 depicts several approaches to shearing along multiple dimensions. A one-dimensional grating can be physically rotated between two positions to acquire time-sequential measurements of the wavefront. Also a pair of orthogonal gratings can produce separated sets of interferograms that can be analyzed by using multiple

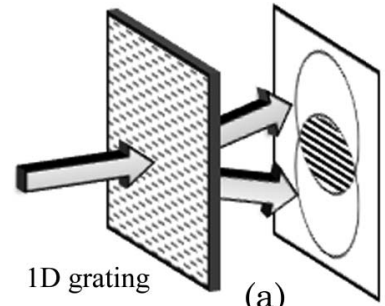

(a)

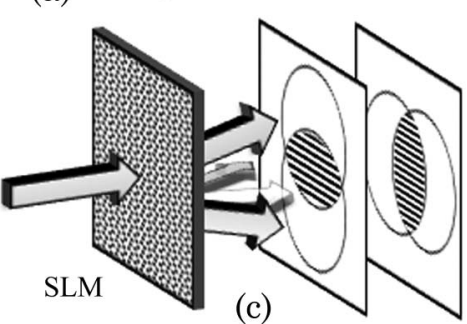

(b)

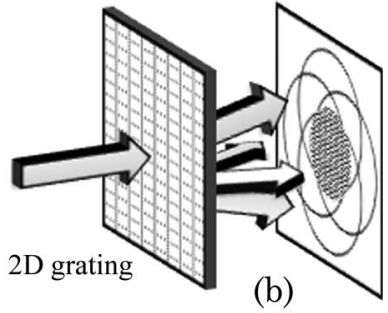

(c)
Fig. 1. Diffractive elements perform LSI by overlapping diffraction orders. (a) Information about the wavefront is obtained along the shearing direction. (b) Two-dimensional gratings simultaneously record the entire field while (c) SLMs sequentially create multiple interferograms.

detectors [7]. Multiorder gratings divide wavefronts into several replicas that simultaneously overlap [8]. The resulting interference provides additional sets of fringes between nonparallel diffraction orders but reduces interferogram contrast and imposes further sampling constraints. Most interesting is an electrically addressable element such as a spatial light modulator (SLM) to display gratings of different periods and orientations. This permits shearing variability within a two-dimensional field. Feedback increases system sensitivity and dynamic range by changing the shear on the basis of characteristics of the wavefront.

The simplest demonstration of an integrated, diffractive interferometer places a fixed grating directly on the surface of an image sensor. This setup provides only one-dimensional shearing; however, it is used to test processing algorithms and identify performance requirements for variable LSI designs. Section 3 details the theory behind diffractive shearing including spectral processing and wavefront reconstruction steps. Preliminary experimental results for a fixed grating device are shown in Section 4. Section $\underline{5}$ highlights the benefits of variable shearing and emphasizes the potential gains realized by substituting a SLM for the fixed grating. Section 6 is dedicated to the physical specifications and requirements of the SLM for an adaptive wavefront sensor. Concluding remarks are made in Section 7.

\section{Description of LSI Method}

The amplitude and phase of a general field are defined by $|f(x, y)| \exp [j \phi(x, y)]$, and a laterally shifted version can be written as $|f(x-s, y)| \exp [j \phi(x-s, y)]$, where $s$ is the magnitude of a one-dimensional shift. The resulting intensity occurring from interference appears as 


$$
\begin{aligned}
g(x, y)= & || f(x, y) \mid \exp [j \phi(x, y)] \\
& +\left.|f(x-s, y)| \exp [j \phi(x-s, y)]\right|^{2}
\end{aligned}
$$

and can expressed in the form

$$
g(x, y)=a(x, y)+b(x, y) \cos [\phi(x, y)-\phi(x-s, y)]
$$

with

$$
\begin{gathered}
a(x, y)=|f(x, y)|^{2}+|f(x-s, y)|^{2}, \\
b(x, y)=2|f(x, y)||f(x-s, y)| .
\end{gathered}
$$

The cosine term in Eq. (2) depends on the relative phase difference between the fields and is the desired quantity for wavefront sensing. This signal is embedded along with other irradiance variations and is typically measured with contour mapping in cases with known reference arms.

Passing the original field through a diffraction grating causes the light to split into multiple orders, each being a copy of the original, tilted at an angle $\theta_{m}$ defined by the grating equation at normal incidence

$$
\sin \theta_{m}=m \frac{\lambda}{d}
$$

where $m$ refers to the diffraction order at wavelength $\lambda$ and grating period $d$. Diffractive replication is useful, since it not only creates lateral separation $s$ when allowed to propagate, but also incorporates a modulation frequency $f_{0}$ within the cosine term. This is due to the linear OPD between the tilted diffraction orders (Fig. 2). The interference intensity between diffraction orders $\alpha$ and $\beta$ is written as

$$
\begin{aligned}
g(x, y)= & a(x, y) \\
& +b(x, y) \cos \left[\phi(x, y)-\phi(x-s, y)+2 \pi f_{o} x\right],
\end{aligned}
$$

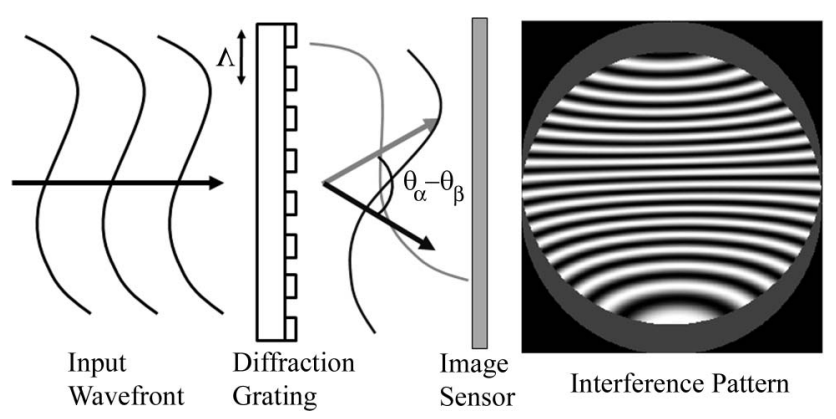

Fig. 2. Diffraction angles create a linear fringe pattern within the interferogram. Wavefront aberrations distort the carrier pattern, which is spectrally processed.

$$
f_{o}=\frac{\sin \left(\theta_{\alpha}-\theta_{\beta}\right)}{\lambda} .
$$

The spatial variation introduced by $f_{o}$ is typically fast compared with the terms in Eqs. (3) and (4) and acts as a carrier frequency for the phase difference signal. Well-separated peaks appear in the spectral domain, allowing the differential phase component to be isolated from other intensity variations [9]. To highlight the phase detection steps, Eq. (6) is rewritten in the form

$$
\begin{aligned}
g(x, y)= & a(x, y)+c(x, y) \exp \left(i 2 \pi f_{o} x\right) \\
& +c^{*}(x, y) \exp \left(i 2 \pi f_{o} x\right),
\end{aligned}
$$

where

$$
c(x, y)=\frac{1}{2} b(x, y) \exp [i \phi(x, y)-i \phi(x-s, y)]
$$

and $*$ denotes the complex conjugate. Fourier transformation of Eq. (8) yields Eq. (10), with capital letters referring to the Fourier spectra and $\omega_{\alpha}$ the spatial frequency in the $\alpha$ direction:

$$
\begin{aligned}
G\left(\omega_{x}, \omega_{y}\right)= & A\left(\omega_{x}, \omega_{y}\right)+C\left(\omega_{x}-f_{o}, \omega_{y}\right) \\
& +C^{*}\left(\omega_{x}+f_{o}, \omega_{y}\right) .
\end{aligned}
$$

Modulation from the tilted wavefronts is seen as shifts in the spectrum, allowing the phase measurement to be isolated with a single sideband spectral filter (Fig. 3). The phase difference signal results in spectral broadening of the modulation peak and affects the width of the required filter.

The carrier may be demodulated by shifting the filtered sideband toward the origin. This is viable only for theoretical, single-frequency carriers and may lead to residual Fourier components in experimental scenarios. In practice, the gradient phase measurement is mixed with a reference interferogram acquired by shearing and overlapping a collimated field [10]. Let $c_{m}(x, y)$ represent the complex signal of the differential phase as seen in Eq. (9) and

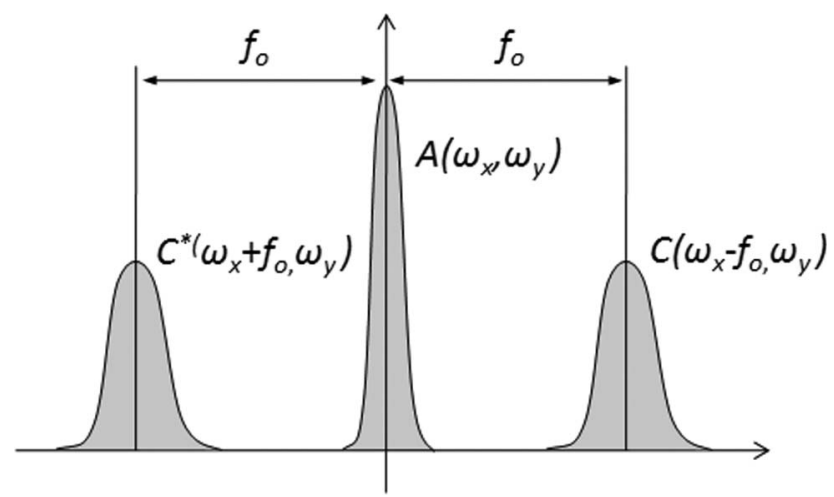

Fig. 3. Carrier modulation allows the phase difference signal to be isolated from other illumination components. The spectral width of the sidebands contains the phase information. 
$c_{r}(x, y)$ correspond to the complex measurement formed from shearing a plane wave as a reference pattern. The phase gradient can be retrieved through the relation

$$
\begin{aligned}
& \Delta \phi_{y}(m, n)=\phi(m, n)-\phi(m, n-s), \\
& m, n=0,1,2, \ldots, N-1,
\end{aligned}
$$

$$
\phi(x, y)-\phi(x-s, y)=\arctan \left[\frac{\operatorname{Re}\left\{c_{r}(x, y)\right\} \operatorname{Im}\left\{c_{m}(x, y)\right\}-\operatorname{Im}\left\{c_{r}(x, y)\right\} \operatorname{Re}\left\{c_{m}(x, y)\right\}}{\operatorname{Im}\left\{c_{r}(x, y)\right\} \operatorname{Im}\left\{c_{m}(x, y)\right\}+\operatorname{Re}\left\{c_{r}(x, y)\right\} \operatorname{Re}\left\{c_{m}(x, y)\right\}}\right]
$$

where Re and Im represent the real and imaginary components, respectively. The additional reference measurement is performed only once for a given carrier. Mixing is superior to spectral translation because it removes all frequencies associated with the carrier, including additional frequencies stemming from illumination aberrations. The signal resulting from Eq. (11) uncovers the differential phase measurement of the field, wrapped from $-\pi$ to $\pi$. Care must be taken during phase unwrapping schemes to avoid streaks emanating from discrete points of residue [11].

The phase difference is the difference between the original field and a sheared copy, thereby representing slope information in the direction of the shear. To analyze an entire two-dimensional wavefront, shearing and analysis must be performed along two nonparallel directions. Multidirectional phase derivatives generated from multilateral shearing produce additional signals that aid in reconstruction accuracy, despite adding complexity to the detection process [8]. Note that the piston term associated with optical aberrations cannot be detected by using LSI. Periodic aberrations with identical frequencies to the carrier are not seen, since no subtraction signal results.

At this point, slope information about the wavefront has been extracted from the modulated interferogram. To estimate the three-dimensional profile of the wavefront itself, the measured signal must undergo spatial integration. Several mathematical algorithms based on least-squares fitting and modal estimation have been proposed for differential wavefront reconstruction [12-15]. Our implemented technique is a generalization based on modal estimation methods proposed by Elster and Weingartner $[16,17]$, which rely on an inverse Fourier filter known as the shearing transfer function. Let the discrete phase difference along each orthogonal direction in an $N \times N$ field be written as

$$
\begin{aligned}
& \Delta \phi_{x}(m, n)=\phi(m, n)-\phi(m-s, n), \\
& m, n=0,1,2, \ldots, N-1,
\end{aligned}
$$

where $s$ is the discrete lateral shear distance in samples. The shift theorem of Fourier transforms states that a translation in space introduces a linear phase shift in frequency [18]:

$$
\operatorname{FT}\left\{\Delta \phi_{x}(m, n)\right\}=\operatorname{FT}\{\phi(x, y)\}\left[1-\exp \left(-\frac{i 2 \pi \omega_{x} s}{N}\right)\right]
$$

$$
\operatorname{FT}\left\{\Delta \phi_{y}(m, n)\right\}=\operatorname{FT}\{\phi(x, y)\}\left[1-\exp \left(-\frac{i 2 \pi \omega_{y} s}{N}\right)\right]
$$

The bracketed exponentials in Eqs. (14) and (15) are known as shearing transfer functions. Dividing the right-hand side by this term isolates the spectral frequencies of the wavefront profile along each shearing direction. The final spatial form of the data is recovered with a simple inverse transform.

The shearing transfer function has an inherent drawback regarding frequency poles occurring at multiples of $N / s$, causing the exponential to become zero. These Fourier components will be lost, but may be replaced by averaging values from adjacent points, i.e., letting $\mathrm{FT}_{x}(m, n)=\left[\mathrm{FT}_{x}(m+1, n)+\right.$ $\left.\mathrm{FT}_{x}(m-1, n)\right] / 2$. Estimating the missing information may result in reconstruction error; however, it is tolerable provided that the shear $s$ does not create an excessive number of leakage points [19].

The concept of integration over the slope measurement leads to one final point of ambiguity regarding the size of the overlapping region relative to the size of the input wavefront. The common area between the wavefront and its sheared copy is of dimension $(N-s) \times N$. The wavefront under test is $N \times N$. This dimensional mismatch hinders the direct application of the shearing transfer function and requires data preprocessing through a periodicity constraint defined by the periodicity inherent to the exponential basis functions of the inverse filter. The idea is known as the natural extension of the difference function, which was obtained in $[16,17]$. 
Periodicity within the differential wavefront occurs when the summation of each row or column has a zero sum. Considering the special case where $s$ is an integer multiple of $N$, the gradient phase may be extended by using

$$
\begin{aligned}
& \Delta \phi_{x}(m, n)=-\sum_{p=0}^{(N / s)-1} \Delta \phi_{x}(m+p s, n), \\
& m=0,1,2, \ldots, s-1, \quad n=0,1,2, \ldots, N-1 ; \\
& \Delta \phi_{y}(m, n)=-\sum_{q=0}^{(N / s)-1} \Delta \phi_{y}(m, n+q s), \\
& n=0,1,2, \ldots, s-1, \quad m=0,1,2, \ldots, N-1 .
\end{aligned}
$$

For the general case when $s$ is not an integer multiple of $N$, similar treatment is still possible by first extending the measurement data beyond the desired $N \times N$ size until the constraint is met. Some data interpolation and smoothing is necessary to suitably meet the above requirement; however, only the solution within the $N \times N$ interval is retained. The added ability to utilize any shear distance provides powerful insight regarding input aberration types and noise relationships, which are explored in Section 5. To summarize the process of wavefront reconstruction from an arbitrary gradient phase measurement, the following steps are necessary:

- Impose periodicity via natural extension so that each row and column of the gradient has zero sum and $N \times N$ dimensions.

- Fourier transform each array of phase differences.

- Perform integration through division with the shearing transfer function.

- View the reconstructed wavefront by using inverse Fourier transformation.

\section{Fixed Diffractive Shearing Experiment}

The integrated LSI consists of a one-dimensional binary phase grating mounted directly onto the surface of a CMOS image sensor. The grating has 42 lines/mm and is designed for $1064 \mathrm{~nm}$ illumination. Shearing is performed by allowing the \pm 1 st diffraction orders to propagate within the $2 \mathrm{~mm}$ thickness of the grating substrate. Binary phase patterns are ideal for LSI because of their strong firstorder diffraction and zero-order suppression.

The diffraction orders overlap at the sensor plane to create $11.2 \mu \mathrm{m}$ carrier fringes. The carrier must be sampled at a rate higher than the Nyquist frequency to avoid aliasing of modulated gradient data. Conversely, dense sampling of the fringe places spectral sidebands close to the origin, requiring a narrow spectral filter that may not encompass all gradient phase spectral components. The carrier should be sampled so as to provide good separation from the origin and appropriate bandwidth on both sides of the spectral peak and avoid aliasing of the modulated data.

A 2 megapixel monochrome CMOS sensor with a $3.2 \mu \mathrm{m}$ pixel pitch is chosen to detect the interference pattern formed on its surface. The phase object under test is a plano-convex lens with a $100 \mathrm{~mm}$ focal length. Though 3.5 pixel sampling per fringe period is within the defined sampling criteria, practical problems prevent the sensor from accurately identifying the carrier and distinguishing small deviations in periodicity. Coherent speckle and moiré are dominant noise sources that corrupt spectral information when few samples span the carrier. Additionally, high-order diffraction gives rise to aliased interference fringes that overlap the carrier frequency, further hindering detection. The rectangular image in Fig. 4(a) shows the interferogram captured by the system in Fig. $4(\mathrm{~b})$, which overfills the sensor array. Poor carrier modulation and moiré are clearly seen in Fig. 4(c), which ultimately damage the sensitive phase information.

The system is modified to include a $3.6 \times$ magnifying $4 \mathrm{~F}$ relay to decrease the diffraction angle and thereby increase the fringe period. The grating is no longer attached to the CMOS sensor, but instead resides within the front focus of the relay. The relay system reduces the dynamic range by shrinking the usable sensor bandwidth. The modifications can be avoided by selecting a grating with a slightly larger pitch or by selecting a higher-resolution sensor to accommodate both sampling and environmental concerns. The modified system is used solely for verification of the processing methods described in Section 3 .

The optical setup in Fig. $\underline{5}$ is used to measure the radius of curvature of the $10 \overline{0} \mathrm{~mm}$ focal length, planoconvex lens. The imager sensor is rotated $60^{\circ}$ with respect to the grating orientation to minimize moiré between the interference pattern and the pixel array.

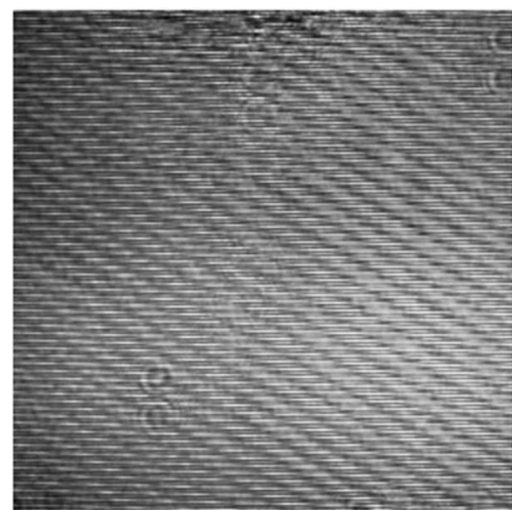

(a)

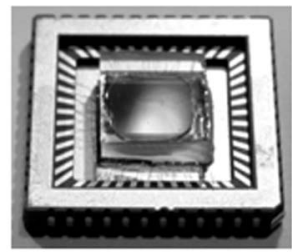

(b)

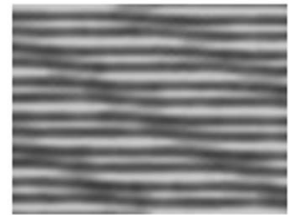

(c)
Fig. 4. (a) Low sampling and moiré disrupt the carrier modulation seen using (b) an integrated device consisting of a fixed phase grating and image sensor. (c) Poor detection of the horizontal carrier fringes is seen in the magnified view. 


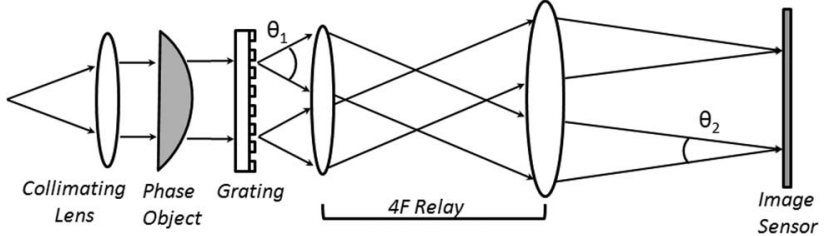

Fig. 5. Integrated LSI is modified by using a $4 \mathrm{~F}$ relay with magnification to reduce the diffraction angle from $\theta_{1}$ to $\theta_{2}$ and increase the carrier period.

The spherical wavefront emanating from the lens is captured, reconstructed, and compared with a surface profile measurement performed using an interference microscope (Fig. 6). The measured radius of curvature differs by $9.2 \%$ and may be attributed to measuring the phase front just after the lens, and not directly at the lens plane. Much of the measurement difficulty stems from the small signal generated from shearing a slowly varying wavefront. Increasing the lateral shift significantly disrupts the carrier periodicity, resulting in larger spectral components that stand out against the background noise. The impact of the shearing ratio is discussed in the following section.

\section{Shearing Ratio}

The lateral shear distance is one adjustable quantity that separates LSI from fixed wavefront sensing techniques such as $\mathrm{SH}$. The shearing ratio is defined as the fractional shift within the normalized field size. Shearing ratios of 0.01 overlap $99 \%$ of the wavefront, while 1.0 creates no common interference area and provides no gradient information.

The shearing ratio governs a trade-off between overlapping field size and magnitude of the differential signal. Large areas of overlap maximize the

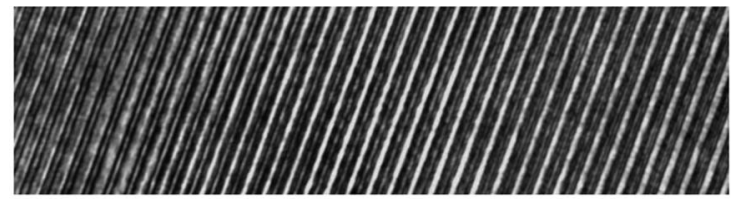

(a)

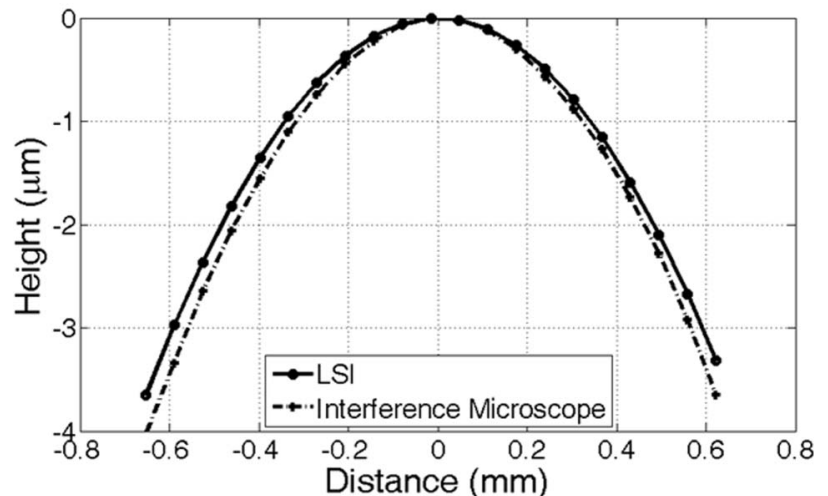

(b)

Fig. 6. (a) A section of the interferogram captured from the modified LSI is used to reconstruct the spherical profile. (b) The lens profile is compared with a phase-shifting microscope. number of data points to create an accurate estimation of the wavefront profile by minimizing data extension. However, since spatial phase variation is typically slow compared with the sample pitch, the resulting difference measurement for small shears is diminutive and difficult to detect, especially in the presence of noise. Large shearing ratios interfere substantially separated field points to yield an increased OPD at the cost of a reduced common area. This scenario requires additional data extension and increases the likelihood of reconstruction error. The significance of altering the shear ratio can be seen visually in Fig. 7 , with small shears producing near periodic fringes, while increasing the shear further disrupts carrier regularity. The distorted fringes in Fig. 7(c) contain more Fourier content, which stands out in noisy environments.

Simulations are performed by using rectangular data sets versus circular ones for geometric simplicity. Sets of Zernike polynomials generate the input phase profile. These are sheared to form synthesized interferograms to process under the algorithm described in Section 3. Two specific wavefronts are simulated, encompassing the cases of slow phase changes, Fig. 8(a), and increased spatial variation, seen in Fig. 8(b). The frequency of spatial variations is associated with certain aberration types. Spherical and coma lead to slow changes, while high-order aberrations cause faster spatial variations.

The spatial autocorrelation of the phase profile is a measure of similarity between the field and shifted versions of itself. Autocorrelation is similar to convolution, except it results in the difference between probability distributions of the signals instead of the sum. The output measurement is always twice

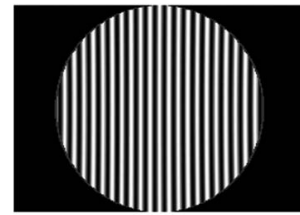

(a)

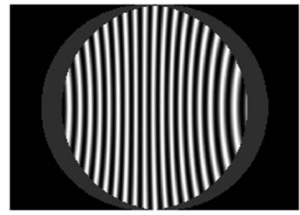

(b)

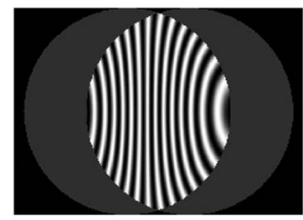

(c)

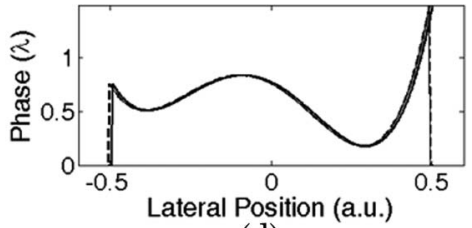

(d)

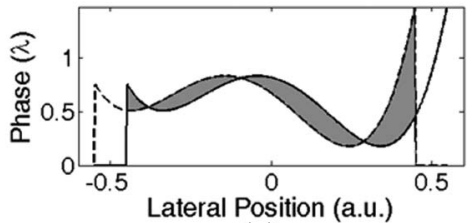

(e)

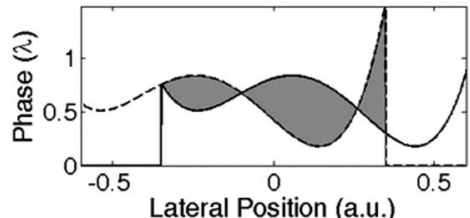

(f)
Fig. 7. OPDs between the sheared points of overlap disrupt fringe carrier periodicity. Adjusting the shearing ratio from (a) 0.01 to (b) 0.1 and (c) 0.3 increases the difference signal highlighted in (d), (e), and (f), respectively. 


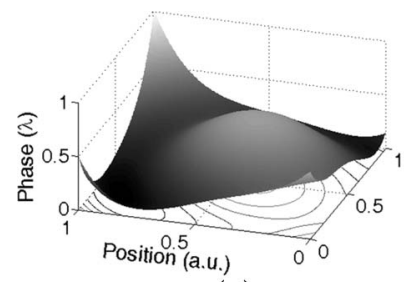

(a)

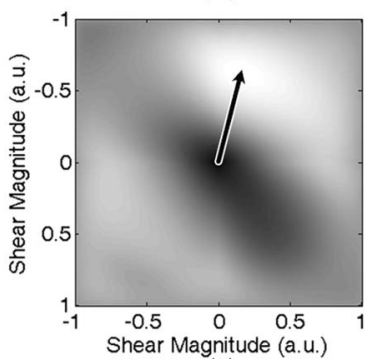

(c)

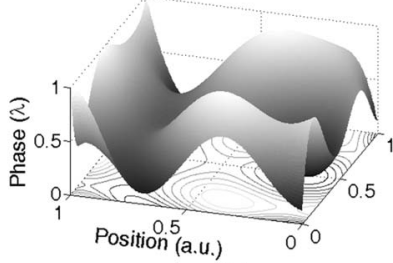

(b)

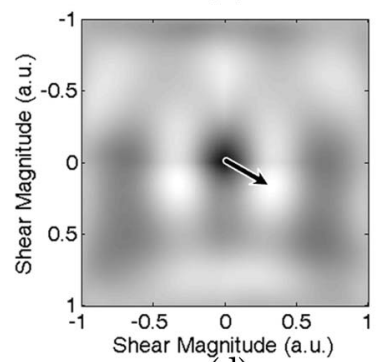

(d)
Fig. 8. Autocorrelation maps identify the shift needed for maximum OPD. (a) Wavefronts exhibiting slow spatial variation produce (c) large difference signals with large shears while (b) increased spatial variation requires (d) smaller shifts.

as large in dimension as the input and is maximal at the origin. Maps of the autocorrelation are seen in Figs. 8(c) and 8(d). The distance between the maximum (origin) and minimum pinpoints the shift that yields the greatest OPD within the wavefront. The magnitude and direction of this shift is highlighted by arrows within the figures. A single shift along the identified direction maximizes the differential signal; however, a pair of shears is needed to span the two-dimensional field. The highlighted vector is broken down into orthogonal shear components along the rows and columns. The synthesized wavefront with slow spatial change has greatest signal from shears of 0.62 and 0.17 along the rows and columns, respectively. Alternatively, shifts of 0.13 and 0.32 along the rows and columns result in the largest phase difference when analyzing the wavefront with increased spatial variation. The autocorrelation map identifies the optimum shearing ratio based only on the wavefront profile, ignoring impacts from data extension and interferogram noise.

To investigate the relationship between shearing ratios and interferogram noise, white Gaussian noise is added to each synthesized interferogram prior to processing. The signal-to-noise ratio ( $\mathrm{SNR}$ ) is computed in decibels as the logarithm of the ratio between normalized fringe intensity and random background noise. The added noise affects the contrast of the interferogram and coincides with spectral frequencies of the differential phase data. The rootmean-square (RMS) error in wavelengths between the final wavefront reconstruction and the known input is used as the figure of merit.

The profile of the reference wavefront seen in Fig. 9(a) is sheared by using equal, orthogonal ratios spanning from 0.01 to 0.7 . Gaussian noise is added to each interferogram to simulate SNRs ranging from 40 to $20 \mathrm{~dB}$. The effect on reconstruction is seen in
Figs. 9(b)-9(d). Decreased SNR causes measurement errors in the gradient, which leads to streaks along each direction of integration. Ripples manifest from combining reconstructions along each direction, adversely affecting the overall smooth profile. SNRs below $10 \mathrm{~dB}$ completely lose details of the wavefront, capturing only a rough estimate of the general profile shape. Simulated SNRs above $30 \mathrm{~dB}$ exhibit very accurate reconstructions with less than $\lambda / 100$ error. The RMS is plotted as a function of shearing ratio for increasing SNR values in Fig. 10(a). The curves identify specific shearing values that yield reconstruction error minimums. At high SNRs, small shearing ratios yield the lowest reconstruction error. Small disruptions in fringe periodicity stand out in the high contrast of the interferogram. As noise levels rise, error minimums initially appear at 0.12 and more significantly at 0.53 shearing ratios. These values are slightly less than the shear optima identified by autocorrelation. Differential signal strength versus overlap area will have diminishing returns on error improvement due to increased data extension. The plots in Fig. 10 are for identical shear values along both orthogonal directions. Allowing independent shear magnitudes along the rows and columns to better model the shift direction identified by autocorrelation lowers the RMS error by $8.2 \%$ at $20 \mathrm{~dB}$.

The second wavefront with increased spatial variation in Fig. 8(b) is simulated under the same shearing and noise conditions. Observing the RMS error versus shearing ratio in Fig. 10(b) yields best reconstructions at shifts between $\overline{0.18}$ and 0.22 . These values are once again in close agreement with those determined from autocorrelation. Including independent shearing along each direction further reduces the RMS error by $2.3 \%$. The optimal shear in each scenario is significantly different, suggesting that reconstruction accuracy exhibits strong depen-

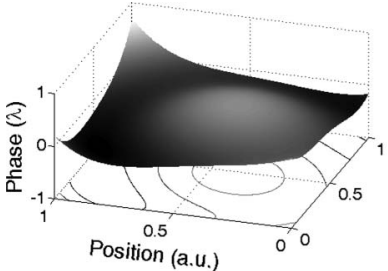

(a)

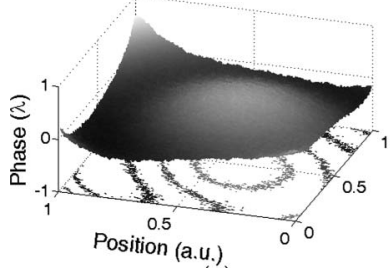

(c)

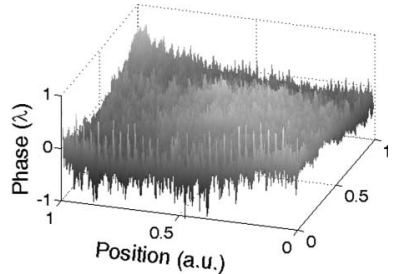

(b)

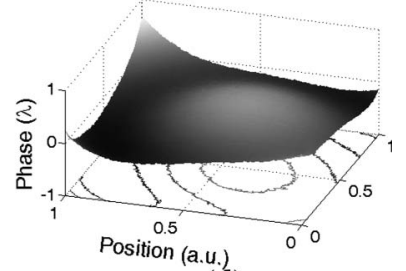

(d)
Fig. 9. (a) Simulated reference wavefront sheared and with Gaussian noise added to the interferogram prior to processing. Reconstructions from (a) $10 \mathrm{~dB}$, (b) $20 \mathrm{~dB}$, and (c) $30 \mathrm{~dB}$ SNRs. A low SNR loses all profile detail, while a high SNR creates reconstructions with $>\lambda / 100$ accuracy. 


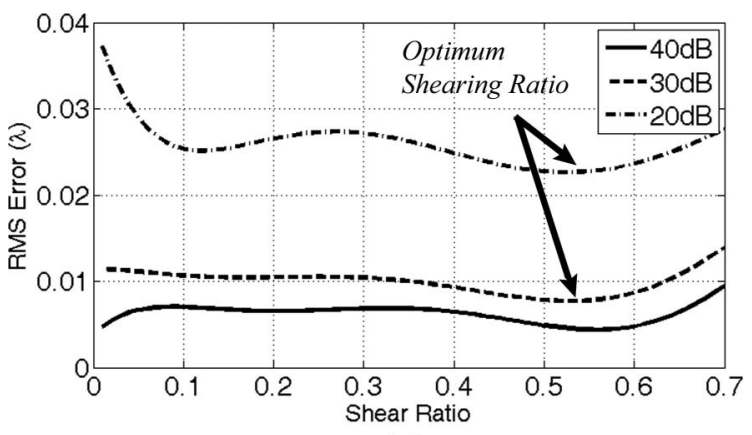

(a)

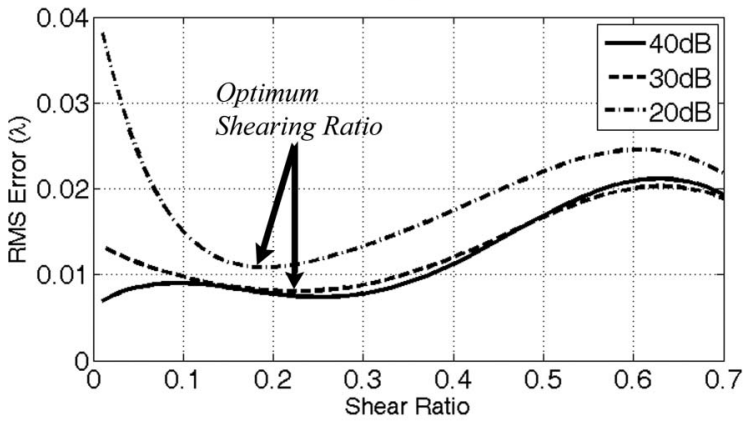

(b)

Fig. 10. Varying the shear ratio at different SNRs leads to reconstruction error minimums that depend on the wavefront profile. (a) Slowly varying wavefronts benefit from large shears, while (b) smaller shears perform best for increased spatial variation.

dence on the type and frequency of the aberrations present. A system that can actively change the shearing ratio in response to different input wavefronts can achieve greater accuracy and dynamic range over a wide variety of potential input profiles.

\section{Variable LSI Sensor}

To create variable lateral shearing, the propagation distance between a fixed grating and sensor can simply be adjusted, allowing the tilted orders to separate. Other implementations vary the rotation angle between a series of crossed gratings [20,21] or rely on rotation within Talbot self-imaging to alter the region of overlap [22]. Mechanical motion and tight tolerances associated with these techniques are not ideal for integrated devices.

Alternatively, liquid-crystal spatial light modulators (SLMs) can act as gratings by displaying binary phase patterns. The grating pitch alters the diffraction angle and affects the shearing ratio when propagated a fixed distance to the image sensor. SLMs have been incorporated into phase shifting interferometry systems $[23,24]$ but are yet to find use as diffractive shearers. The main reason is the discrete pixel pitch, which determines the change in grating frequency and ultimately the realizable shift in shearing ratio.

The diffraction angle varies with the sine of the grating frequency as indicated by Eq. (5) in Section 3 . Small shearing ratios arise from larger grating periods to reduce wavefront tilt, while greater shifts rely on smaller periods. The discrete pitch of the SLM also governs the step size between shearing ratios. A very small pitch is needed for small shearing increments, as well as to generate larger diffraction angles needed in high-shearing scenarios. A general system consisting of a $5 \mathrm{~mm}$ field propagating $10 \mathrm{~mm}$ to the sensor, calls for a $5 \mu \mathrm{m}$ SLM pitch simply to switch between ratios of 0.3 and 0.4. To achieve higher shearing ratios and increased resolution pushes the pitch closer to $1 \mu \mathrm{m}$. Additionally, the pitch of the image sensor is of concern to properly sample interferograms with increased carrier frequencies created from larger diffraction angles.

Devices with very small pitches are not currently available as commercial products. High-resolution SLMs with $8 \mu \mathrm{m}$ pixels are used in diffractive beam splitting; however, they utilize reflective, liquid-crystal-on-silicon technology [25]. Transmission-based SLMs are better suited for integrated LSI devices, but have pixels higher than $25 \mu \mathrm{m}$. Increasing the propagation distance between the diffractive element and sensor relaxes the pitch specification by decreasing the need for high diffraction angles to create large shearing ratios. This approach affects the incremental step size between shearing ratios, resulting in coarse shifts that may not yield the type of variability desired for detecting high-order aberrations.

Fabrication and packaging improvements need to be made in order to create addressable phase modulators with pitches below $5 \mu \mathrm{m}$ at high fill factors to promote diffraction efficiency. Combining such a device with a high-resolution image sensor creates an integrated wavefront sensor with the unique ability to adapt its shearing parameters based on the incoming phase. Future techniques may involve shearing within localized regions of a field to provide unprecedented levels of dynamic range. The process for identifying the optimum shearing ratio of an unknown wavefront may involve preliminary shear measurements and is currently a topic of further investigation.

\section{Conclusion}

We have designed and simulated an integrated, lateral shearing interferometer comprising a binary phase grating and CMOS image sensor. Tilt between overlapping diffraction orders creates a carrier fringe within the interferogram that provides gradient slope information. Fourier-based processing is used to detect and reconstruct the phase profile of the input field.

The magnitude of the lateral shear determines the points of overlap between the wavefront copies and directly affects the optical path difference within the interferogram. Variable shearing enables the LSI technique to adjust to different types of wavefront aberration and minimizes reconstruction error. The SNR and its connection to shearing ratio has been explored with a clear relationship to the autocorrelation of the input. The optimum shear differs 
depending on the spatial variation of the wavefront and noise levels present during detection.

A system incorporating a SLM as a variable diffractive element can adapt the shearing ratio for maximum sensitivity and dynamic range by changing the diffraction angle. Consequently, no transmission modulator technology currently offers the $5 \mu \mathrm{m}$ resolution needed for the desired shear increment. The added flexibility would allow this LSI system to detect high-order aberrations and avoid resolution-dynamic-range trade-offs inherent to other integrated wavefront sensors.

The authors acknowledge Ronald Stack (Distant Focus Corporation) and Tessera Digital Optics Technologies for providing equipment and technical assistance. This research was supported by the Defense Advanced Research Projects Agency (DARPA) via the MONTAGE program, grant HR0011-04-I-0045.

\section{References}

1. K. Kinnstaetter, A. W. Lohmann, J. Schwider, and N. Streibl, "Accuracy of phase shifting interferometry," Appl. Opt. 27, 5082-5089 (1988).

2. J. Primot, "Theoretical description of Shack-Hartmann wavefront sensor," Opt. Commun. 222, 81-92 (2003).

3. P. S. Fairman, B. K. Ward, B. F. Oreb, D. I. Farrant, Y. Gilliand, C. H. Freund, A. J. Leistner, J. A. Seckold, and C. J. Walsh, "300-mm-aperture phase-shifting Fizeau interferometer," Opt. Eng. 38, 1371-1380 (1999).

4. V. Ronchi, "Forty years of history of a grating interferometer," Appl. Opt. 3, 437-451 (1964).

5. P. Hariharan, Optical Interferometry, 2nd ed. (Academic, 2003).

6. G. Paez, M. Strojnik, and G. Torales, "Vectorial shearing interferometer," Appl. Opt. 39, 5172-5178 (2000).

7. J. C. Wyant, "Double frequency grating lateral shear interferometer," Appl. Opt. 12, 2057-2060 (1973).

8. S. Velghe, J. Primot, N. Guerineau, M. Cohen, and B. Wattellier, "Wave-front reconstruction from multidirectional phase derivatives generated by multilateral shearing interferometers," Opt. Lett. 30, 245-247 (2005).

9. M. Takeda, H. Ina, and S. Kobayashi, "Fourier-transform method of fringe-pattern analysis for computer-based topography and interferometry," J. Opt. Soc. Am. 72, 156-160 (1982).
10. S. De. Nicola and P. Ferraro, "Fourier transform method of fringe analysis for moiré interferometry," J. Opt. A 2, 228-233 (2000).

11. D. Bone, "Fourier fringe analysis: the two-dimensional phase unwrapping problem," Appl. Opt. 30, 3627-3632 (1991).

12. D. Fried, "Least-square fitting a wave-front distortion estimate to an array of phase-difference measurements," J. Opt. Soc. Am. 67, 370-375 (1977).

13. R. Hudgin, "Wave-front reconstruction for compensated imaging," J. Opt. Soc. Am. 67, 375-378 (1977).

14. K. Freichlad and C. Koliopoulos, "Modal estimation of a wave front from difference measurements using the discrete Fourier transform," J. Opt. Soc. Am. 3, 1852-1861 (1986).

15. L. Poyneer, D. T. Gavel, and J. M. Brase, "Fast wave-front reconstruction in large adaptive optics systems with use of the Fourier transform," J. Opt. Soc. Am. A 19, 2100-2111 (2002).

16. C. Elster and I. Weingartner, "Solution to the shearing problem," Appl. Opt. 38, 5024-5031 (1999).

17. C. Elster and I. Weingartner, "Exact wave-front reconstruction from two lateral shearing interferograms," J. Opt. Soc. Am. A 16, 2281-2285 (1999).

18. J. Goodman, Introduction to Fourier Optics, 3rd ed. (Roberts \& Company, 2005).

19. P. Liang, J. Ding, Z. Jin, C. Guo, and H. Wang, "Two-dimensional wave-front reconstruction from lateral shearing interferograms," Opt. Express 14, 625-634 (2006).

20. M. P. Rimmer and J. C. Wyant, "Evaluation of large aberrations using a lateral-shear interferometer having variable shear," Appl. Opt. 14, 142-150 (1975).

21. A. Lohmann and O. Bryngdahl, "A lateral wavefront shearing interferometer with variable shear," Appl. Opt. 6, 1934-1937 (1967).

22. S. Yokozeki and T. Suzuki, "Shearing interferometer using the grating as the beam splitter," Appl. Opt. 10, 1575-1580 (1971).

23. Y. Bitou, "Digital phase-shifting interferometer with an electrically addressed liquid-crystal spatial light modulator," Opt. Lett. 28, 1576-1578 (2003).

24. S. Zhao and P. S. Chung, "Digital speckle shearing interferometer using a liquid-crystal spatial light modulator," Opt. Eng. 45, 105606 (2006).

25. A. Hermerschnidt, S. Krüger, and G. Wernicke, "Binary diffractive beam splitters with arbitrary diffraction angles," Opt. Lett. 32, 448-450 (2007). 


\section{Queries}

1. Re. "but have pixels higher than $25 \mu \mathrm{m}$," do you mean "a pitch higher than..." or "pixels larger than..."? 